\title{
The very high spatial resolution infrared thermography on ITER-like tungsten monoblocks in WEST Tokamak
}

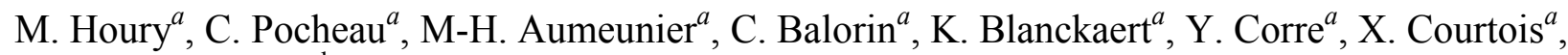

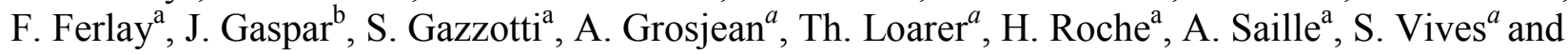 \\ the WEST Team
}

\author{
${ }^{a}$ CEA-IRFM, F-13108 Saint-Paul-Lez-Durance, France \\ ${ }^{b}$ Aix-Marseille Université, CNRS, IUSTI UMR 7343, 13013 Marseille, France
}

\begin{abstract}
A new Infrared diagnostic has been developed by CEA-IRFM and installed in the WEST tokamak to measure surface temperature of the actively cooled W-monoblocks components as foreseen for the ITER Divertor, with a very high spatial resolution of $100 \mu \mathrm{m}$. The goals are to investigate the effects of the shaping of these components on the heat load deposition pattern, the evolution of pre-damaged components specifically introduced in WEST, the behavior of the leading edges regarding the assembling tolerances between adjacent monoblocks, and finally to contribute to the specification assessment of the ITER divertor units. In WEST, each Plasma Facing Unit is composed of $35 \mathrm{~W}$-monoblocks of individual surface of $28 \times 12 \mathrm{~mm}$. To analyze heat load pattern and phenomena on such tiny surfaces, the leading edges and in the narrow gaps between monoblocks $(400-500 \mu \mathrm{m})$, a $100 \mu \mathrm{m}$ spatial resolution is required. Then, a Very High spatial Resolution (VHR) infrared diagnostic has been specially developed at CEA-IRFM. The VHR operates at $1.7 \mu \mathrm{m}$ wavelength to take advantage of the dynamic of the signal for the temperature range $\left(400\right.$ to $\left.3600^{\circ} \mathrm{C}\right)$. The VHR infrared diagnostic is now operational above the divertor sector made of actively cooled $\mathrm{W}$-monoblocks and graphite inertial components with $\mathrm{W}$ coating. This paper gives a description of the diagnostic.
\end{abstract}

Keywords: ITER, Plasma facing components Protection, Diagnostics, infrared, tungsten monoblocks.

\section{Introduction and context}

The ITER full tungsten (W) divertor will be made of several hundred thousands of $\mathrm{W}$ monoblocks with surface of a few $\mathrm{cm}^{2}$ exposed to the plasma. Assembled on a pipe like a rosary for the cooling by a water loop, the requirement for the mechanical assembly tolerance is highly demanding [1]. Indeed, due to the low angle of incidence of the magnetic field lines, on the surface of the components, to spread the heat loads, a misalignment of the components can induce heat loads on the side of the component with an incidence close to perpendicular (Fig. 1) [2].
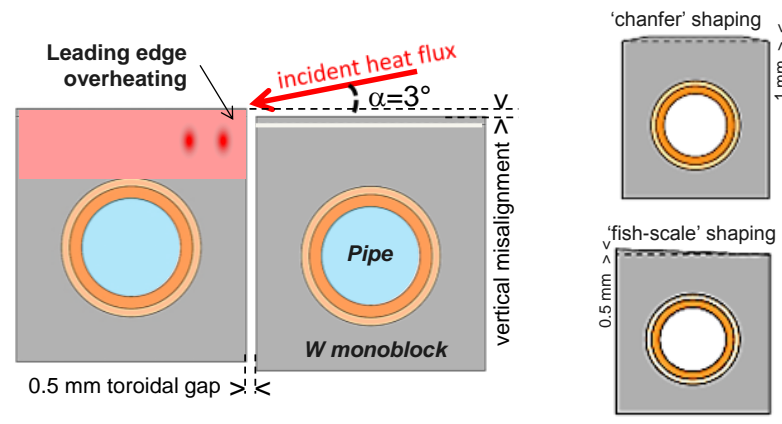

Fig. 1: Cross section of the ITER-like Plasma Facing Unit $(P F U)$, showing the impact of a misalignment between neighbouring PFU in terms of local overheating of the protruding leading edge (left), and proposed monoblock shaping options to handle this issue (right).
The heat loads deposited per unit of area can then lead to a local melting of components and irreversible damages [3], [4]. Designs are proposed to reduce the risk induced by misalignments. They consist of a machining of component surface for a shadowing effect over the successive components or a chamfer on the edges of the components ("fish-scale" shaping, Fig. 1) or a chamfer on the edges of the components ("chamfer shaping, Fig. 1). The misalignment tolerance for the components assembly and the capability of the applied designs to reduce the risk of damage, as well as the impact of damages on the heat exhaust capacity of the components are crucial issues for ITER [5].

With the modification of the Tore Supra tokamak into WEST, the CEA-IRFM offers a platform to test the concept and designs of the Plasma Facing unit (PFU) made of Tungsten monoblocks for ITER [6], [7]. PFUs of different manufacturers and different design and shaping will thus be tested under different plasma conditions and with ITER-relevant heat loads deposition ranging from $10 \mathrm{MW} / \mathrm{m}^{2}$ up to $20 \mathrm{MW} / \mathrm{m}^{2}$ of peak heat flux.

The observation of phenomena related to heat loads on the leading edges of the components is a challenge. Indeed, phenomena occur on very narrow surfaces and with tricky angles and distances for observation purposes. To this end, a Very High spatial Resolution (VHR) infrared diagnostic has been specially developed at CEA-IRFM to observe heat loads on the ITER-like PFU to be tested in WEST. This paper describes the 
diagnostic design, the performances obtained as well as the first observations performed in WEST.

\section{Description of the diagnostic}

\subsection{Tokamak environment}

The WEST divertor with the ITER-like PFUs is a conical surface at the bottom of the vacuum vessel. The observation on the divertor is made from an upper port. The line of sight between the first mirror and the divertor is about $2 \mathrm{~m}$, with an incidence close to $90^{\circ}$ (Fig 2).

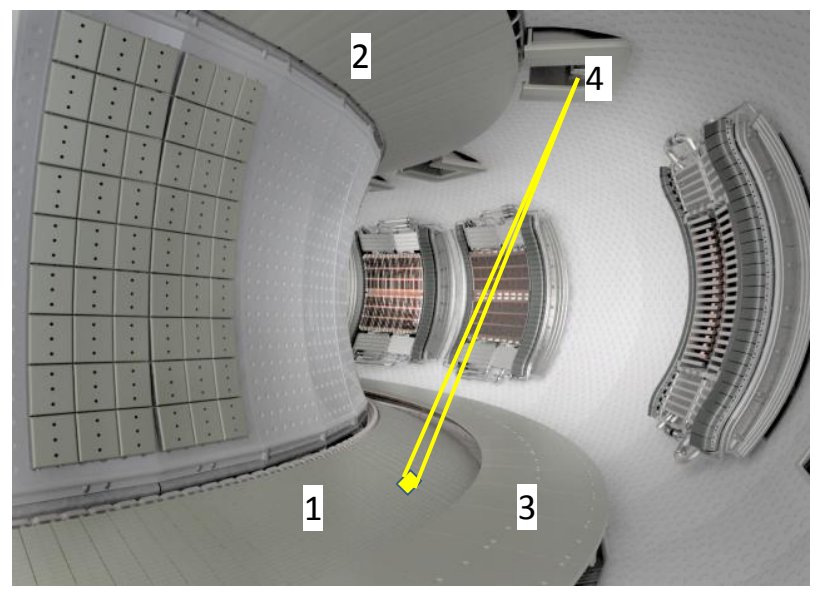

Fig 2: CAD view of the WEST Vessel. (1) lower divertor (2) upper divertor, (3) Baffle, (4) upper port with VHR diagnostic.

The head of the endoscope and the first mirror are positioned $30 \mathrm{~cm}$ backward in the port to prevent the heat load deposition from particles and radiative flux. Thus, it was not necessary to install a water cooling system on the head of the endoscope, significantly reducing the design and integration constraints.

\subsection{Optical design}

The gaps between the monoblocks $(0.5$ to $1 \mathrm{~mm})$, the chamfers (1 $\mathrm{mm}$ range), the misalignments between PFU (maximum of $0.3 \mathrm{~mm}$ ) and the local overheating expected on few mm led to a requirement for the spatial resolution of $100 \mu \mathrm{m}$, in order to provide enough measurement points for analysis.

The observation of the components with a pixel resolution of $0.1 \mathrm{~mm}$ constrains the objects and image fields of the optical design. The observed field is $64 \mathrm{~mm}$ x $51 \mathrm{~mm}$ with a matrix of $640 \times 512$ pixels with a pitch of $15 \mu \mathrm{m}$, thus defining a transverse magnification of $1 / 6.67$.

To achieve this high spatial resolution, the design of the IR diagnostic is made of 6 mirrors and 4 lenses (Fig 3): 1 spherical mirror (\#3), a cylindrical mirror (\#4), 4 lenses and 4 flat mirrors (\#1, \#2, \#5, \#6); the first 2 can be remotely moved to scan the observable surface. Mirrors of $10 \mathrm{~cm}$ diameter are silicon with protected $\mathrm{Au}-$ coating.

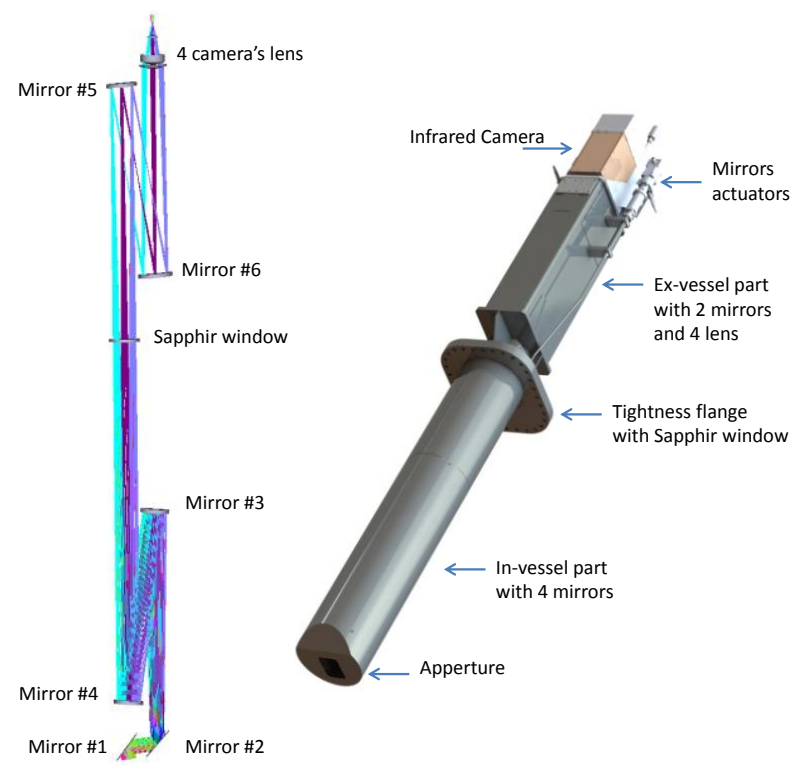

Fig 3: Design of the optical path (left part) CAD view of the VHR IR diagnostic (right part).

\subsection{Mechanical design}

The conceptual mechanical design of the endoscope is inspired by space industry. The endoscope structure is light, compact and "flexible-stiff" to sustain transient events such as disruptions. Modal vibrational frequency calculations have been performed to ensure that, in the vibratory environment of the tokamak, the structure of the endoscope could not resonate. Thus, this design does not present vibratory mode below $50 \mathrm{~Hz}$. The part under vacuum consists of successive platforms for the installation of mirrors, connected by tie rods and flexible structures. A stainless steel cylinder cover encloses the assembly without thermal contact with the internal structure (Fig 3 and 4). The dimension of the endoscope from one end to the other is $2.33 \mathrm{~m}$; the diameter of the in-vessel part is $200 \mathrm{~mm}$

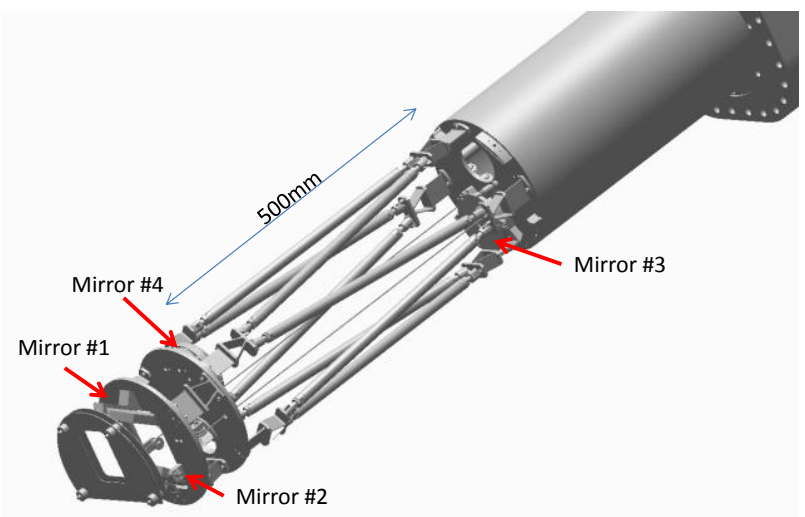

Fig 4: Design of the mechanical structure holding the mirrors (in-vessel part of the endoscope).

Due to the ripple effect and the displacement of the strike points on the divertor, the field of view, which is quite narrow $(64 * 51 \mathrm{~mm})$, must be able to scan the divertor surface to reach the areas of interest. The two head mirrors are thus actuated to rotate on their axis of rotation (poloidal for the mirror 1 and toroidal for the 
mirror 2). The field of view can thus move on the divertor on a surface of $360 \times 420 \mathrm{~mm}$, covering 14 PFUs (toroidal direction) and 28 monoblocks (poloidal direction). The actuators are located at the upper end of the endoscope (away from the magnetic field) and are used to move cables for the rotation of the two first mirrors to move the field of view on the divertor (Fig 3). The displacement of the field of view on the selected monoblocks is automated using dedicated software.

Due to the conical shape of the divertor, the line of sight distance varies according to the observed field of view $( \pm 4 \mathrm{~cm})$. This is optically compensated by a motorized camera lens which allows adjusting the focus according to the observed area on the divertor.

\subsection{Infrared camera}

The infrared camera of the VHR IR diagnostic is one of the 12 homemade WEST infrared cameras (Fig. 5) developed for the real time monitoring and protection of WEST components [8].

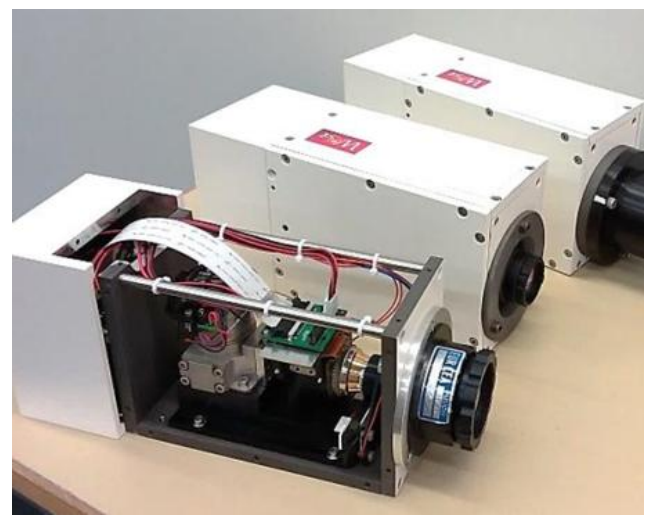

Fig 5: WEST Home-made cameras with soft iron magnetic shield.

The sensor is an InSb array which operate in the spectral range $[1.5-5.0 \mu \mathrm{m}]$, with a pixel frame of $640 \times 512$ at $250 \mathrm{~Hz}$ acquisition frame rate. A soft iron magnetic shielding protects the camera components against the magnetic fields of the tokamak. An actively cooled plate is fixed with screws on the casing to maintain a stable temperature. The band-pass filter installed few $\mathrm{mm}$ in front of the sensor is also cooled using a Peltier module to reduce the contribution of the background.

The bad pixels replacement, the correction of nonuniformity of the detector and the digital level to temperature conversion is performed in real time in an external FPGA board.

\section{Performances}

\subsection{Temperature Measurement}

Temperature calibration has been performed on CEA-IRFM test benches with a black body up to $1600^{\circ} \mathrm{C}$ with a point source and $500^{\circ} \mathrm{C}$ with an extended source. The extrapolation to temperature up to $3000^{\circ} \mathrm{C}$ has been performed based on the good linearity of the sensor. The optical transmission of the endoscope has been measured at $55 \%$. Emissivity remains a delicate point for absolute temperature measurement with metallic components. We use emissivity values from measurements performed on a dedicated test bed. Tungsten emissivity has been measured versus surface temperature, wavelength and surface state of the monoblocks (mainly its roughness). In addition, sensors embedded in the components of the divertor (thermocouples and Fiber Bragg Grating) contribute by cross-measurement to reducing the uncertainty on the emissivity and the surface temperature. The choice of the $1.7 \mu \mathrm{m}$ wavelength for the optimization of the measurement also offers an improvement on the absolute error on the temperature (compare to middle wavelength range). The choice of this wavelength is based on the very large temperature range $\left(400\right.$ to $\left.3600^{\circ} \mathrm{C}\right)$ foreseen for the experimental studies (up to melting of the leading edges).

\subsection{Image Resolution}

The modulation transfer function (MTF) is a basic performance measurement of an imaging system describing the optical response of the system as a function of the spatial frequencies. The experimental MTF in the Figure 6 (bottom) has been derived from a single tilted slit target (Line Spread Function) using a Fourier Transform procedure. The slit width has been fixed to $25 \mu \mathrm{m}$ much smaller compared to the width of

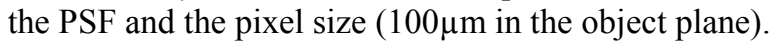

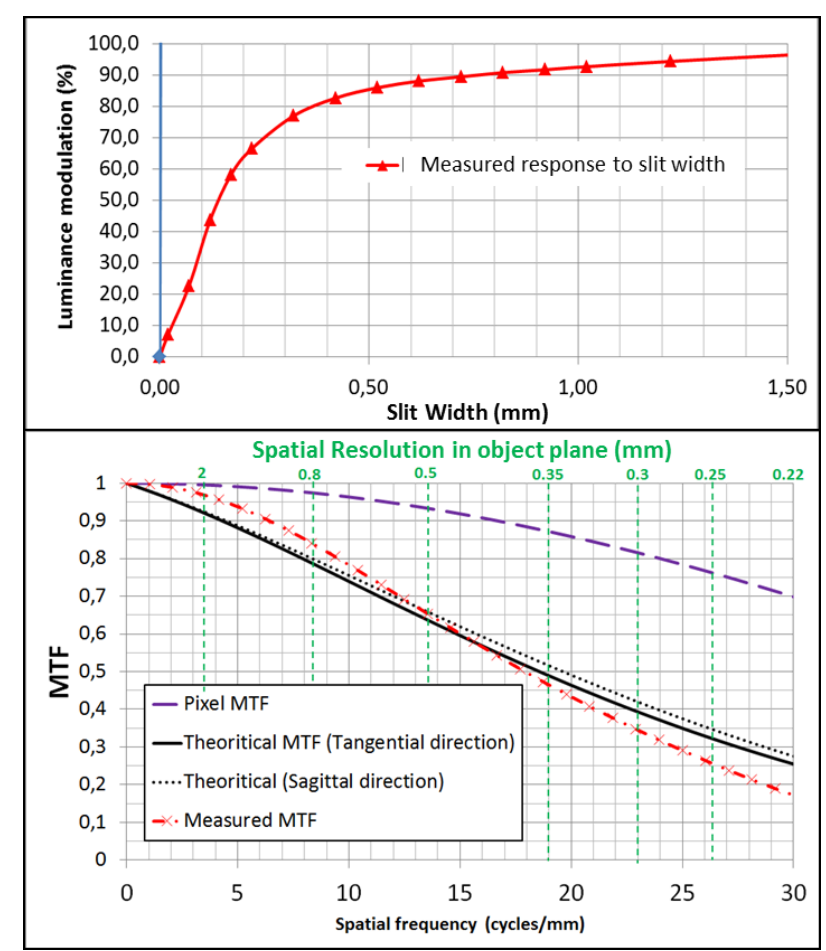

Fig 6: Slit response function (upper figure) and modulation transfer function (bottom figure) of the VHR diagnostic.

The Figure 6 shows the measured MTF and a comparison with the pixel MTF (with a pixel of $0.1 \mathrm{~mm}$ in object plane).The response of our system is here limited by the optics (mainly by the diffraction phenomenon). We note that at $300 \mu \mathrm{m}$, the MTF is $35 \%$ which is a commonly used lowest value for imaging 
contrast acceptance. Fig 6 (upper) shows the slit response function (SRF) obtained by measuring the luminance ratio at a given (thin) slit width with a very wide slit width [9].

\subsection{Observation on WEST components}

During the first WEST campaign where the VHR IR diagnostic was installed (summer 2018), the heat loads on the ITER like PFU were not sufficient to reach the temperature threshold of the VHR. However, images have been collected during events producing a lot of radiation such as disruptions. The figure 7 is an image obtained during a disruption (pulse \#52205). The IR rays coming from the surface of the monoblocks are reflection, but not thermal emission of the monoblock. We clearly see the monoblocks of 2 ITER-like PFUs and on the right side an inertial PFU with $\mathrm{W}$ coating. The gaps and chamfers are clearly observed around the surfaces of the monoblocks, showing that the VHR IR system is able to monitor the submillimeter features of the ITER like PFU as required.

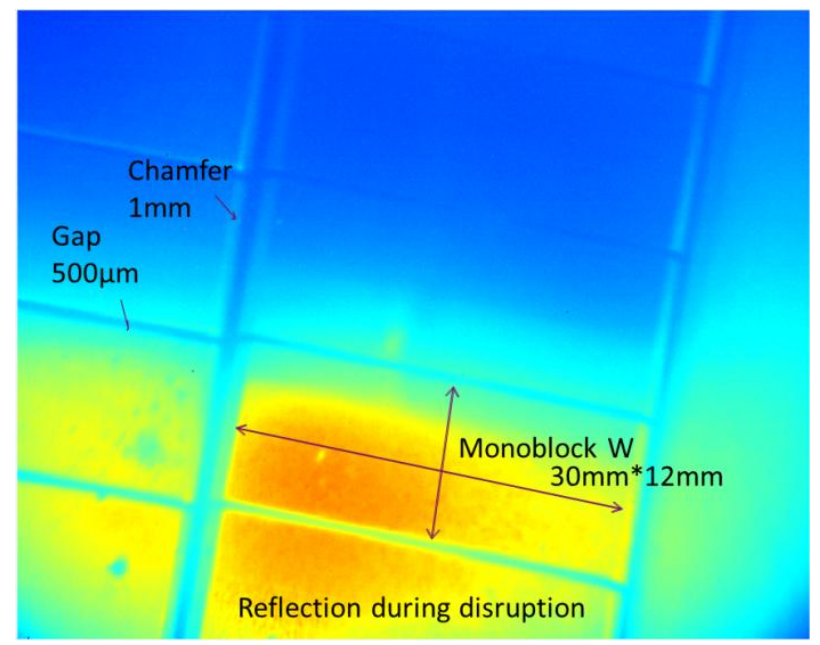

Fig 7: Image of the surface of the Plasma Facing Unit on lower Divertor during disruption (Color scale in arbitrary units).

\section{Conclusion}

The VHR infrared diagnostic on WEST is dedicated to observation of heat loads depositions on ITER-like PFUs. The pixel resolution is $100 \mu \mathrm{m}$ and then imaging spatial resolution at MTF- $35 \%$ is $300 \mu \mathrm{m}$ leading to a surface temperature error of $15 \%$ at $1000^{\circ} \mathrm{C}$.

The diagnostic is operational and follows the power ramp-up of WEST, ready for observation of heat loads on leading edges and effects from PFU misalignments and components surface shaping.

Future upgrades of the diagnostic are being studied. They concern (i) the implementation of a two-color measurement for a better accuracy on the measurement of temperature and (ii) fast measurement for the observation of fast thermal transient events such as the ELM energy deposition.

\section{Acknowledgments}

"This work has been carried out within the framework of EUROfusion Consortium and has received funding from Euratom research and training programme. The views and opinions expressed herein do not necessarily reflect those of the European Commission.

\section{References}

[1] S. Carpentier et al, 2014 Phys. Scr. T159 014002

[2] Y. Corre, et al, Nucl. Fusion 57 (2017) 016009.

[3] J.P. Gunn et al, Nucl. Fusion 57 (2017) 046025

[4] R. Dejarnac et al, Nucl. Fusion 58 (2018) 066003

[5] R.A.Pitts et al., Journal of Nuclear Materials, volume 438, 2013, S48

[6] J. Bucalossi, et al, Fusion Eng. Des. 89 (2014) 907-912.

[7] D. Guilhem, et al, Phys. Scr. 167 (2016) 014066.

[8] X. Courtois et al, Fusion Eng. Des. (2018), SOFT conference

[9] X. Courtois et al, Fusion Eng. Des. In Press, doi 10.1016/j.fusengdes.2018.05.042 